\title{
Renoprotective Effects of Gallic Acid Against Gentamicin Nephrotoxicity Through Amelioration of Oxidative Stress in Rats
}

Hassan Ahmadvand ${ }^{1,2}$

https://orcid.org/0000-0002-9406-3592

\author{
Negar Nouryazdan ${ }^{1}$ \\ https://orcid.org/0000-0002-5166-8647
}

Maryam Nasri ${ }^{3}$

https://orcid.org/0000-0002-6857-6132

\section{Glavizh Adibhesami ${ }^{3}$}

https://orcid.org/0000-0003-1794-6006

\section{Esmaeel Babaeenezhad 4* \\ https://orcid.org/0000-0003-2549-7802}

\begin{abstract}
${ }^{1}$ Lorestan University of Medical Sciences, Faculty of Medicine, Department of Clinical Biochemistry, Khorramabad, Lorestan, Iran; ${ }^{2}$ Lorestan University of Medical Sciences, Razi Herbal Medical Research Center, Faculty of Medicine, Khorramabad, Lorestan, Iran; ${ }^{3}$ Lorestan University of Medical Sciences, Department of Biochemistry and Genetics, Khorramabad, Lorestan, Iran; ${ }^{4}$ Shahid Beheshti University of Medical Sciences, School of Medicine, Student Research Committee, Department of Clinical Biochemistry, Tehran, Tehran, Iran.
\end{abstract}

Received: 2020.03.04; Accepted: 2020.06.01.

${ }^{*}$ Correspondence: es.babaeenezhad1391@gmail.com; Tel.: +98-916-8499013 (E. Babaeenezhad)

HIGHLIGHTS

- Gallic acid ameliorates gentamicin-induced nephrotoxicity.

- Gallic acid decreases levels of NO and MDA and enhances abilities of GSH, GPX, and CAT.

- Gallic acid improves altered liver and renal function markers in nephrotoxic animals.

- Gallic acid reduces renal histopathological injuries.

Abstract: Gallic acid (GA), as a strong antioxidant, was selected in this study to investigate its possible nephroprotective effects against gentamicin (GM)-induced nephrotoxicity. Twenty-four rats were separated into three groups ( $n=8)$ : group 1 (control group) received saline $(0.5 \mathrm{~mL} /$ day), group 2 (GM group) received GM (100 mg/kg/day), and group 3 (treated group) received GM (100 mg/kg/day) and GA (100mg/kg/day). All treatments were performed intraperitoneally for 12 days. After 12 days, the rats were euthanized, and kidneys were removed immediately. For serum preparation, blood samples were collected before killing. Kidney paraffin sections were prepared from one of the kidneys and stained by the periodic acid-Schiff process. GA significantly decreased GM-induced renal histopathological injuries, including tubular necrosis, tubular cast, and leucocyte infiltration compared with the GM group. Additionally, GA significantly improved proteinuria, serum levels of urea and creatinine, and serum activities of aspartate aminotransferase (AST) and alanine 
aminotransferase (ALT) compared with nephrotoxic animals. Furthermore, GA caused a significant improvement in the levels of cholesterol (Chol), low-density lipoprotein (LDL), high-density lipoprotein (HDL), and cardiac risk ratios 1 and 2 in comparison with nephrotoxic animals. GA administration was observed to significantly improve the levels of lipid peroxidation, nitric oxide (NO), and glutathione (GSH) compared with the GM group. Finally, the activities and gene expression levels of catalase (CAT) and glutathione peroxidase (GPX) significantly increased following GA administration compared with the GM group. Our results indicated that GA has potential protective effects against GM nephrotoxicity by reducing oxidative stress in rats.

Keywords: gentamicin nephrotoxicity; gallic acid; oxidative stress; lipid profile; renal; liver function markers.

\section{INTRODUCTION}

Gentamicin (GM) is recognized as an effective aminoglycoside antibiotic and commonly applied in treating infections induced by gram-negative bacteria. Different studies have indicated that ototoxicity, hepatotoxicity, and nephrotoxicity are the most important side effects of GM. Among these complications, nephrotoxicity is the main problem that can inhibit the broad utilization of GM in clinic [1]. Despite the presence of nephrotoxicity in $10-25 \%$ of therapeutic courses [2], the clinical use of GM is still ongoing because of its rapid bactericidal action, broad-spectrum activity, chemical stability, clinical effectiveness, and importantly low-cost [3]. The detail about mechanisms of GM-induced nephrotoxicity are still completely unknown. Different studies have proposed several mechanisms, including oxidative stress, apoptosis, tubular necrosis, phospholipidosis, increased endothelin I, and leukocyte infiltrations [2,4]. GM is absorbed by renal tubular cells through anion transportation system. GM accumulation in these cells finally leads to morphological alterations, functional impairments, and the increase of reactive oxygen species (ROS) and reactive nitrogen species (RNS) in the kidney [5]. During nephrotoxicity, these free radicals promote inflammatory process, apoptosis, and necrosis [2]. The free radicals also suppress renal antioxidant system via protein oxidation [6] and lipid peroxidation (LPO) [7].To solve this problem in recent years, different studies suggested that application of natural antioxidants seems to be a suitable approach to protect the kidney against GM nephrotoxicity [8]. In addition, it has been reported that low levels of antioxidants accelerate nephrotoxicity process [9]. Therefore, studies aiming to find new natural antioxidants with beneficial effects on GM nephropathy are still important.

Nowadays, phenolic compounds are the most important natural antioxidants, which contribute to decrease the incidence of oxidative stress-related diseases [10]. Among the phenolic compounds, gallic acid (GA) (3, 4, 5-trihydroxy benzoic acid) (Figure 1) as an aromatic composition is well-found in many plants such as gallnuts, sumac, tea leaves, and oak bark [11]. GA is documented as a potent antioxidant, free radical scavenger, and an inhibitor of LPO [12]. Indeed, the antioxidant property of GA is associated with the location of hydroxyl groups and other functional groups [12]. Furthermore, other fundamental characteristics of GA can be mentioned, such as anti-bacterial, anti-viral, anti-inflammatory, and anti-cancer [13]. GA and its derivatives are extensively utilized in food industries to prevent LPO induced rancidity [14]. The findings acquired from some studies revealed that GA could protect the kidney against different pathological conditions such as renal ischemia-reperfusion (RIR), chronic kidney disease (CKD), and heavy metalinduced renal damages [15,16]. GA-mediated nephroprotective effects are associated with its abilities to neutralize a variety of free radicals and enhance the potency of endogenous antioxidant system [17].

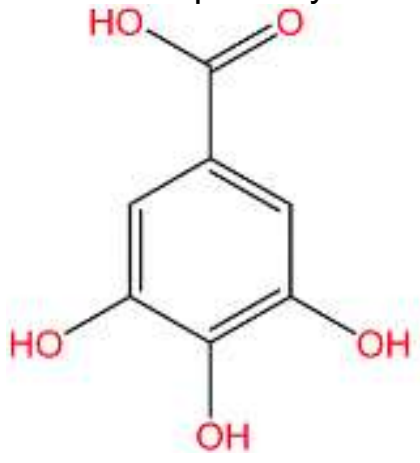

Figure 1. Chemical structure of Gallic acid (3,4,5-Trihydroxybenzoic acid or $\mathrm{C} 6 \mathrm{H} 2(\mathrm{OH}) 3 \mathrm{COOH})$ 
Toxicological studies previously revealed that GA at a dose of $119 \mathrm{mg} / \mathrm{kg} / \mathrm{day}$ had no-observed-adverseeffect level (NOAEL) in male rats [18]. Furthermore, the beneficial effects of the $100 \mathrm{mg} / \mathrm{kg}$ dose of GA against RIR were observed in our recent study [19]. In this study, we investigated the possible effects of GA (100 $\mathrm{mg} / \mathrm{kg}$ ) against GM nephrotoxicity. For this purpose, we designed a histopathological, biochemical, and molecular study.

\section{MATERIAL AND METHODS}

\section{Chemicals}

Tris-Ethylenediaminetetraacetic acid (Tris-EDTA), Tris-HCl and EDTA were obtained from Merck Company (Germany). Commercial kits for the assessment of triglyceride (TG), cholesterol (Chol), high density lipoprotein cholesterol (HDL-C), urea, creatinine ( $\mathrm{Cr}$ ), alkaline phosphatase (ALP), aspartate aminotransferase (AST), and alanine aminotransferase (ALT) were purchased from Pars Azmoon Company (Tehran, Iran). Trizol reagent and GA (purity: 97.5\%) were obtained from Sigma Aldrich Company (USA). SYBER Green qPCR Master Mix 2x and cDNA synthesis kit were purchased from Yekta Tajhiz Azma Company (Iran). GM was provided from Alborz Darou Company (80 mg/2mL, AMP).

\section{Animals and Experimental Design}

Twenty-four adult male Wistar rats (180-200 g) were prepared from the Razi Herbal Medicines Research Center in Lorestan, Iran. The rats were housed in wire-bottomed cages with standard conditions including an adjusted temperature of $25 \pm 1^{\circ} \mathrm{C}$, a humidity of $50 \pm 10 \%$, and 12 hours: 12 hours:12 hours' light: dark cycle. The Animal Ethics Committee of Lorestan University of Medical Sciences approved our study protocol. This research was also consistent with the guidelines of the National Health and Medical Research Council.

The rats were randomly divided into three equal groups $(n=8)$ as follows:

Group I (Control group): The rats in this group received intraperitoneal saline $(0.5 \mathrm{~mL} /$ day) for 12 days.

Group II (GM group): The rats in this group received intraperitoneal GM (100 mg/kg/day) [20] for 12 days.

Group III (Treated group): The rats in this group received intraperitoneal GM (100 mg/kg/day) + GA (100 $\mathrm{mg} / \mathrm{kg} / \mathrm{day}$ ) [19] for 12 days. The study was reviewed and approved by the Lorestan University of Medical Science's Ethics Committee (code: LUMS.REC.1397.160).

\section{Sample Collection}

After 12 days of the experiment, all of the rats were housed in individual metabolic cages for the collection of 24-hour urine. 24-hour urine samples were centrifuged for $5 \mathrm{~min}$ (1400 rpm), and the supernatant was collected for the determination of the proteinuria excretion rate. Later, the rats were anesthetized with ketamine ( $75 \mathrm{mg} / \mathrm{kg}$ intraperitoneally) and xylazine $(13 \mathrm{mg} / \mathrm{kg}$ intraperitoneally). Subsequently, blood samples were collected from the heart of each rat and then centrifuged at $3000 \mathrm{rpm}$ for 15 minutes $\left(4^{\circ} \mathrm{C}\right)$ to serum separation. Serum samples were stored at $-20^{\circ} \mathrm{C}$ for further biochemical measurements. Furthermore, the right kidney was separated carefully and fixed immediately in neutral formaldehyde (10\%) for the histopathological evaluations. The left kidney was divided into two equal parts. One of these parts was homogenized immediately in ice-cold phosphate-buffered saline (PBS, $\mathrm{pH}$ 7.4). After that, the homogenate was centrifuged at $18,000 \times \mathrm{g}\left(4^{\circ} \mathrm{C}\right)$ for $30 \mathrm{~min}$; the supernatant was utilized for the assessments of renal biochemical parameters. For the evaluation of mRNA expression levels of antioxidant enzymes, the other part was posited in the Trizol reagent (Sigma-Aldrich Company, USA) and kept at $-70{ }^{\circ} \mathrm{C}$.

\section{Kidney Histopathological Assessment}

For histopathological evaluations, fixed tissue samples with formaldehyde solution (10\%) were processed in a series of graded ethanol and embedded in paraffin. Then, paraffin sections were prepared at $5 \mu \mathrm{m}$ thickness and stained with periodic acid Schiff (PAS) staining method. For the evaluation of the histopathological alterations, different parameters including leukocyte infiltration, tubular cast, and tubular necrosis were observed in 40 fields of proximal convoluted tubules (PCT) of each kidney at a light microscopic magnification of $\times 400$. These histopathological alterations were measured semiquantitatively according to Caramel method [21] as follows:

$0=$ no damage, $1=$ mild, $2=$ moderate (damage $<25 \%)$, 3=marked $(25 \%<$ damage $<50 \%)$, 4=massive (damage $>50 \%$ ). 


\section{Biochemical Assessment}

\section{Measurement of renal function markers}

The markers of kidney function, including serum creatinine $(\mathrm{Cr})$ and urea, were measured by an autoanalyzer (Olympus AU-600, Tokyo, Japan) using commercial kits (Pars Azmoon Company, Tehran, Iran). In order to evaluation of proteinuria excretion rate, total protein in the urine samples was measured turbidimetrically based on Shahangian and coauthors method $[22,23]$.

\section{Measurement of Liver Enzymes Activity}

The activities of liver enzymes in serum including alkaline phosphatase (ALP), aspartate aminotransferase (AST), and alanine aminotransferase (ALT) were executed by above commercial kits and auto-analyzer similar to our previous study [24].

\section{Measurement of lipid profile and atherogenic indices}

The serum levels of triglyceride (TG), cholesterol (Chol), and high-density lipoprotein-cholesterol (HDLC) were determined by an auto-analyzer (Olympus AU-600, Tokyo, Japan) using commercial kits (Pars Azmoon, Tehran, Iran). Moreover, the low-density lipoprotein (LDL) and very-low-density lipoprotein (VLDL) levels were evaluated in our study using Friedewald and coauthors Equation [25]. Ikewuchi [26,27] Equation was utilized for the calculation of cardiac risk ratio 1 (CRR 1): (TC/HDL-C) and CRR 2: (LDL/HDL-C).

\section{Measurement of oxidative stress biomarkers}

Serum and renal malondialdehyde (MDA) levels

The levels of MDA, as the marker of LPO in the serum and kidney of the rats, were measured based on thiobarbituric acid (TBA) assay [28,29]. The absorbance was measured spectrophotometrically at $532 \mathrm{~nm}$.

Serum and renal glutathione (GSH) levels

The measurement of serum and renal levels of GSH was performed spectrophotometrically at $412 \mathrm{~nm}$, according to Ellman's method [30].

\section{Serum and renal glutathione peroxidase (GPX) activities}

Glutathione peroxidase (GPX) activities in the serum and kidney were evaluated according to Rotruck and coauthors method [31]. The absorbance was measured at $420 \mathrm{~nm}$ by an ELISA reader, and GPX activity is shown as $\mathrm{U} / \mathrm{mg}$ protein.

\section{Serum and renal catalase (CAT) activities}

Sinha method [32] was used for the measurement of serum and kidney activities of CAT. The reaction was initiated through the addition of a sample (20 $\mu \mathrm{L}$ of serum or supernatant) in $2 \mathrm{~mL}$ of hydrogen peroxide $\left(\mathrm{H}_{2} \mathrm{O}_{2}, 30 \mathrm{mM}\right)$ in potassium phosphate buffer $(50 \mathrm{mM}, \mathrm{pH} 7.0)$. Enzyme units were considered as $\mathrm{mM}$ of consumed $\mathrm{H}_{2} \mathrm{O}_{2}$ per min $\mathrm{g}$ or $\mathrm{mL}$.

\section{Serum paraoxonase 1 (PON 1) activity}

Serum PON 1 activity was measured by the utilization of paraoxon as a substrate similar to our previous study [33].

Serum nitric oxide (NO) level

Serum NO level in the rats of three groups was measured by evaluation of the nitrite as the end product of NO. The evaluation of the nitrite level was performed based on Giustarini and coauthors method [34].

\section{RNA Isolation and Quantitative Real-Time RT-PCR}

The mRNA expression levels of the antioxidant enzymes, including Cu-Zn SOD, CAT, and GPX, were determined by quantitative real-time reverse-transcription polymerase chain reaction (RT-PCR). Total RNA was extracted from kidney samples with Trizol reagent (Sigma Aldrich Company, USA), as explained 
completely in our previous study [35]. After that, RNA integrity and purity were determined by NanoDrop spectrophotometer (Biochrom WPA Biowave II, UK) and electrophoresis (2\% agarose gel). Following RNA isolation, cDNA synthetization was carried out from $2 \mu \mathrm{g}$ of RNA samples applying cDNA synthesis kit (YT4500, Yekta Tajhiz Azma, Iran) based on its manufacturer's guidelines. The expression levels of target genes (GPX, CAT, and Cu-Zn SOD) and reference gene ( $\beta$-actin) were assessed by RT-PCR using SYBER Green qPCR Master Mix 2x (YT2551, Yekta Tajhiz Azma, Iran). All reactions were performed in triplicate on Rotor-Gene 6000 (Corbett Research) with thermal cycling condition as follows: 1 cycle of $95{ }^{\circ} \mathrm{C}$ for 3 mins and 40 cycles of $95^{\circ} \mathrm{C}$ for 5 secs (denaturation), and 40 cycles of $60^{\circ} \mathrm{C}$ for 20 secs (annealing \& extension). The results of quantitative real-time RT-PCR were analyzed by the $2^{-\triangle \Delta C T}$ method. The sequence of primers (Forward and Reverse) for each gene is indicated in Table 1.

Table 1. The sequence of utilized primers for quantitative real-time RT-PCR

\begin{tabular}{ccl}
\hline Gene & Primer position & \multicolumn{1}{c}{ Primers sequences $\left(\mathbf{5}^{\prime} \rightarrow \mathbf{3}^{\prime}\right)$} \\
\hline SOD $(\mathbf{C u}-\mathrm{Zn})$ & Forward & TTCGAGCAGAAGGCAAGCGGTGAA \\
& Reverse & AATCCCAATCACACCACAAGCCAA \\
\hline \multirow{2}{*}{ GPX } & Forward & GGTGTTCCAGTGCGCAGAT \\
\multirow{2}{*}{ CAT } & Reverse & TCGAACCCGATATAGAAGCCCT \\
\hline \multirow{2}{*}{$\boldsymbol{\beta}$-actin } & Forward & ATTGCCGTCCGATTCTCC \\
& Reverse & CCAGTTACCATCTTCAGTGTAG \\
\hline & Forward & TATCGGCAATGAGCGGTTCC \\
& Reverse & AGCACTGTGTTGGCATAGAGG \\
\hline
\end{tabular}

\section{Statistical Analysis}

All of the parameters were calculated as mean \pm standard error (SE) and compared between the groups using one-way ANOVA followed by LSD test (for histopathological and biochemical parameters) and Dunnett T3 test (for real-time RT-PCR results). Statistical analyses were accomplished by SPSS statistics software (SPSS; version 20). Statistically, the significance between different groups was accepted at a $P$-value $<0.05$.

\section{RESULTS}

\section{Histopathological Results}

The results of the histopathological examination of PAS stained kidney sections from each group are represented in Figures 2 . The induction of nephrotoxicity by 12 days of GM administration could significantly enhance the intensity of tubular necrosis and the levels of tubular cast formation and leukocyte infiltration in the GM group against the control group. By contrast, the examination of kidney sections from animals received 12 days of $\mathrm{GA}+\mathrm{GM}$ showed a significant reduction $(53.43 \%, 59.16 \%$, and $59.58 \%$, respectively) in these histopathological parameters and a marked improvement in renal architectures compared to the GM group (Figure 2).

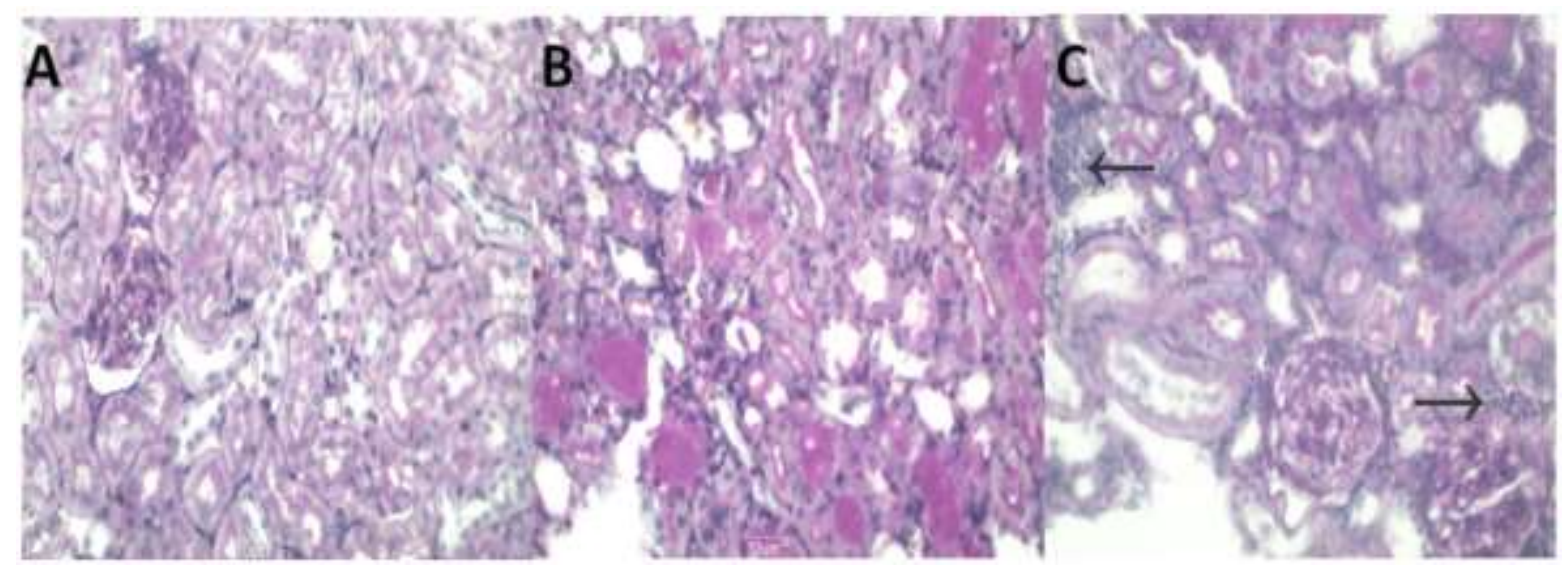

Figure 2. The effects of GA on GM-induced histopathological changes observed in the photomicrographs of kidney sections stained with periodic acid Schiff (PAS). A) A kidney section from the control group indicates normal glomeruli and tubules (x 400). B) A section from the kidney tissue of rats treated with GM shows massive tubular casts, tubulorrhexis, and leukocyte infiltration (x 400). C) A kidney section from GM + GA group represents the amelioration of renal tissue with few casts, a few leukocytes (arrows), and desquamated tubules (x 400). 


\section{Biochemical Results}

\section{The effect of GA on renal function markers in rats with GM nephrotoxicity}

The serum levels of $\mathrm{Cr}$ and urea and proteinuria excretion rate significantly increased (4.03-fold, 3.54fold, and 2.84-fold, respectively) after 12 days of GM administration in the GM group compared with the control group (Figure 3). Treatment with GA was able to decrease $(37.19 \%, 56.30 \%$, and $38.72 \%$, respectively) $\mathrm{Cr}$ and urea levels as well as proteinuria excretion rate in the $\mathrm{GM}+\mathrm{GA}$ group compared to the GM group. GA treatment could not only completely inhibit GM related kidney dysfunction when compared to the control group, but also could reverse GM adverse effects on the renal function when compared to the GM group (Figure 3).

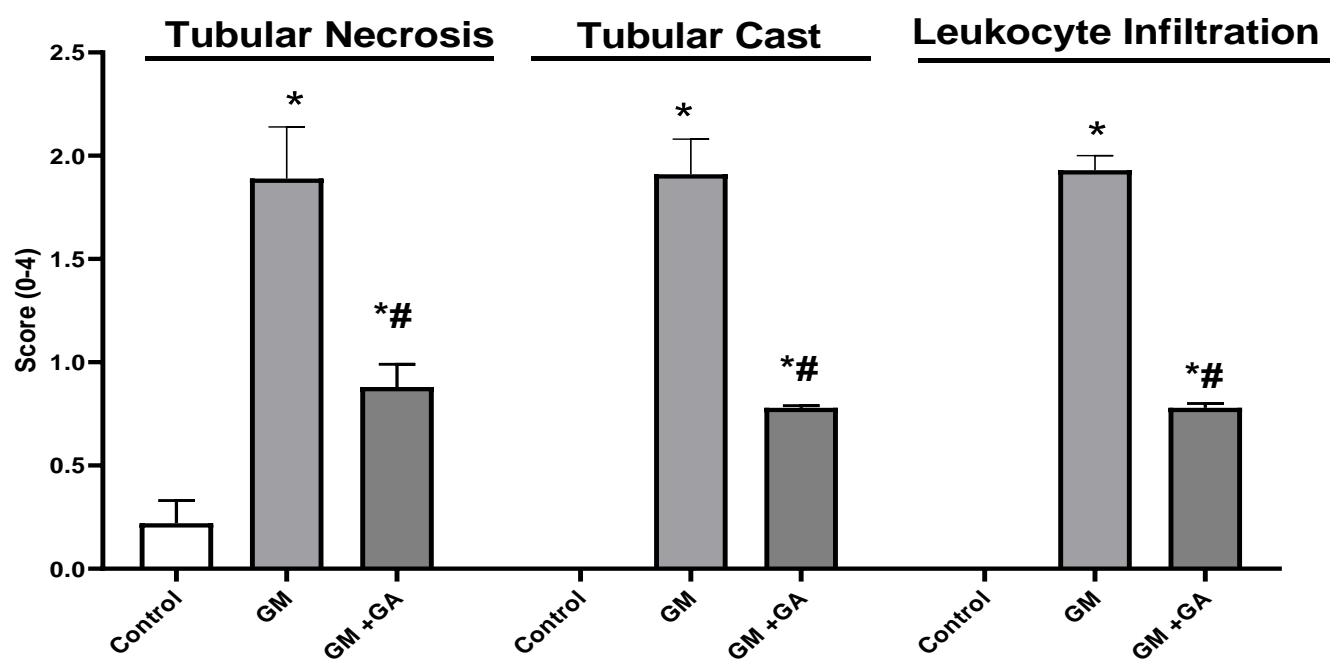

Figure 3. The effects of GA on GM induced renal histopathological changes observed in kidney sections, including tubular necrosis, tubular cast, and leucocyte infiltration in rats with GM nephrotoxicity. Bars display mean \pm SEM. Oneway ANOVA followed by a post hoc LSD test was applied for comparison between groups. ${ }^{*} P<0.05$ as compared with the control group. ${ }^{*} P<0.05$ as compared with the GM group.

\section{The effect of GA on the serum activities of liver enzymes in rats with GM nephrotoxicity}

For evaluation of GA effects on GM induced hepatotoxicity, the activities of AST, ALT, and ALP were determined in the serum of rats. Gentamicin hepatotoxicity, as shown by the significant increment in the serum activities of AST, ALT, and ALP (2.01-fold, 1.67-fold, and 1.62-fold, respectively) was observed in nephrotoxic rats when compared to control rats (Figure 4D, E, and F). By contrast, GA administration could ameliorate GM induced hepatotoxicity, reflected by the reduced serum activities of these enzymes $(54.69 \%$, $46.99 \%$, and $8.82 \%$, respectively) in the GM + GA group compared to the GM group. However, the decrease in ALP activity was not statistically significant $(P=0.33)$. The activities of AST and ALT in GA treated rats reached a normal status in the control group (Figure 4D, E, and F). 


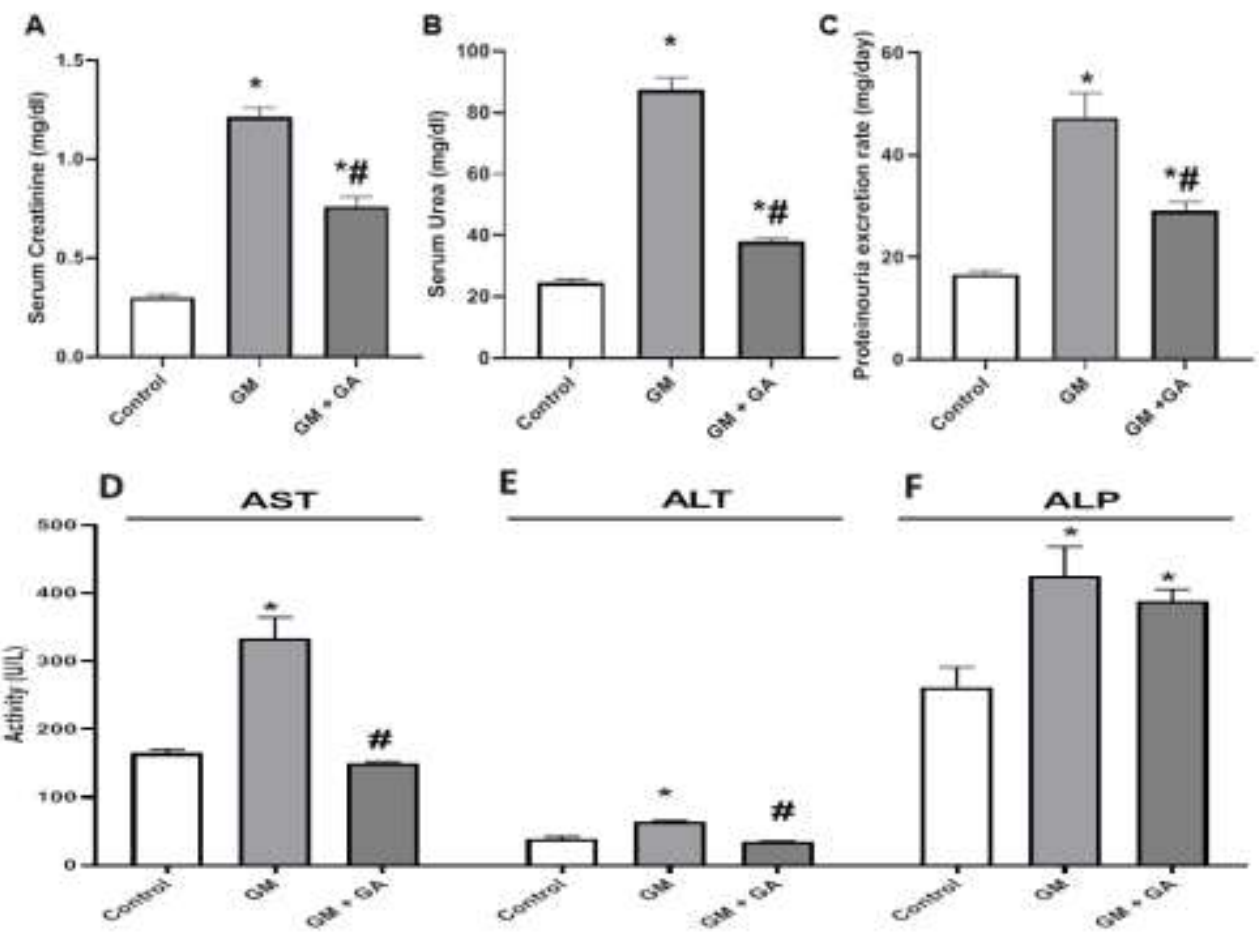

Figure 4. The effects of GA administration on renal and liver function markers in the serum of rats with $G M$ nephrotoxicity, including creatinine $(A)$, urea $(B)$, proteinuria excretion rate $(C)$, AST (D), ALT (E), and ALP (F). Bars represent mean \pm SEM. One-way ANOVA followed by a post hoc LSD test was applied for comparison between groups. ${ }^{*} P<0.05$ as compared to the control group. $\# P<0.05$ as compared with the GM group.

The effect of GA on serum PON1 activity, lipid profile, and atherogenic indices in rats with GM nephrotoxicity

As shown in Table 2, the serum activity of PON1 significantly (2.49-fold) reduced in the GM group compared with the control group. GA treatment enhanced (18.65\%) serum PON1 activity in the treated group in comparison to the GM group, but this change was not statistically significant $(P=0.69)$.

The levels of TG, Chol, LDL, VLDL, CRR1, and CRR2 (1.91-fold, 1.37-fold, 3.40-fold, 1.91-fold, 1.56fold, and 4-fold, respectively) were significantly higher in nephrotoxic rats than in control animals. At the same time, parameters as mentioned above were less $(18.78 \%, 21.18 \%, 68.57 \%, 18.78 \%, 25.26 \%$, and $70 \%$, respectively) in GA treated nephrotoxic rats than in untreated nephrotoxic rats (Table 2). Of note, there was no statistically significant difference in the levels of TG and VLDL between the GA treated group and the GM group ( $P=0.07$ and $P=0.07$, respectively) (Table 2). Furthermore, the serum level of the HDL-C significantly (1.13-fold) decreased in the GM group compared to the control group. By contrast, treatment with GA could significantly increase the HDL-C level (5.98\%) in the GM + GA group against the GM group (Table 2).

Table 2. The effects of GA on serum PON1 activity, the serum levels of lipid profile and NO, and CRRs in GM nephrotoxicity in rats.

\begin{tabular}{llll}
\hline \multicolumn{1}{c}{ Parameters } & \multicolumn{1}{c}{ Control } & \multicolumn{1}{c}{ GM } & \multicolumn{1}{c}{ GM + GA } \\
\cline { 2 - 4 } & \multicolumn{1}{c}{ Mean \pm SE } & \multicolumn{1}{c}{ Mean \pm SE } & \multicolumn{1}{c}{ Mean \pm SE } \\
\hline PON1 activity (nmol/min/mL) & $100.32 \pm 11.44$ & $40.26 \pm 8.88^{*}$ & $47.77 \pm 15.87^{*}$ \\
\hline TG (mg/dL) & $75.83 \pm 4.47$ & $145.3 \pm 13.56^{*}$ & $118.0 \pm 12.05^{*}$ \\
\hline Chol (mg/dL) & $69.83 \pm 1.83$ & $96.00 \pm 5.16^{*}$ & $75.66 \pm 0.95 \#$ \\
\hline VLDL (mg/dL) & $15.16 \pm 0.89$ & $29.06 \pm 2.71^{*}$ & $23.60 \pm 2.41^{*}$ \\
\hline LDL (mg/dL) & $7.43 \pm 1.34$ & $25.30 \pm 6.58^{*}$ & $7.95 \pm 2.98 \#$ \\
\hline HDL-C (mg/dL) & $47.23 \pm 0.47$ & $41.62 \pm 0.45^{*}$ & $44.11 \pm 0.62^{*} \#$ \\
\hline CRR1 (TC/HDL-C) & $1.47 \pm 0.03$ & $2.30 \pm 0.11^{*}$ & $1.71 \pm 0.02^{*} \#$ \\
\hline CRR2 (LDL/HDL-C) & $0.15 \pm 0.02$ & $0.60 \pm 0.15^{*}$ & $0.18 \pm 0.06 \#$ \\
\hline NO (nmol/dL) & $1.27 \pm 0.00092$ & $1.29 \pm 0.00706^{*}$ & $1.28 \pm 0.00378^{*} \#$ \\
\hline N
\end{tabular}

Note: *Significant change in comparison with the control group at $P<0.05$; \#Significant change in comparison with GM group at $P<0.05$; GA: Gallic acid; TG: Triglyceride; Cho: Cholesterol; HDL-C: High-density lipoprotein-cholesterol; LDL: Low-density lipoprotein; VLDL: Very low-density lipoprotein; PON1: Paraoxonase 1; CRR: Cardiac risk ratio; NO: Nitric oxide. 
The effect of GA on serum and renal oxidative stress biomarkers in rats with GM nephrotoxicity

As represented in Figure 5, the serum and renal activities of antioxidant enzymes CAT and GPX significantly decreased (1.72-fold, 2.76-fold, 1.51-fold, and 1.52-fold, respectively) in GM intoxicated rats compared to control rats. Treatment with GA could increase substantially $(52.40 \%, 129.48 \%, 14.63 \%$, and $48.86 \%$, respectively) the activities of these antioxidant enzymes in both kidney and serum so that the serum and renal activities of CAT and the renal activity of GPX reached to the normal activities observed in control rats.

The serum and kidney levels of MDA and GSH and the serum level of NO are depicted in Figure 5. After GM administration of the rats for 12 days, serum and kidney MDA levels and serum NO levels were significantly (2.25-fold, 1.68-fold, and 1.01-fold, respectively) enhanced in the GM group against the control group, however serum and kidney GSH levels were significantly (1.86- fold and 1.66-fold, respectively) decreased. GA administration was able to significantly reduce the enhancement in serum and kidney MDA levels and serum NO levels that followed after 12 days of GM administration (54.34\%, 25.25\%, and $0.77 \%$, respectively). GA treatment in the treated group could significantly enhance only serum GSH level (44.60\%), but not its kidney level, in treated rats in comparison to GM toxicated rats.

A

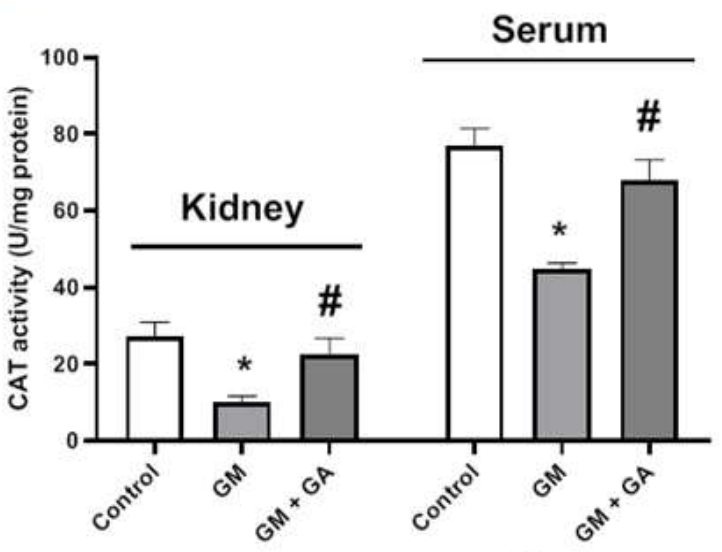

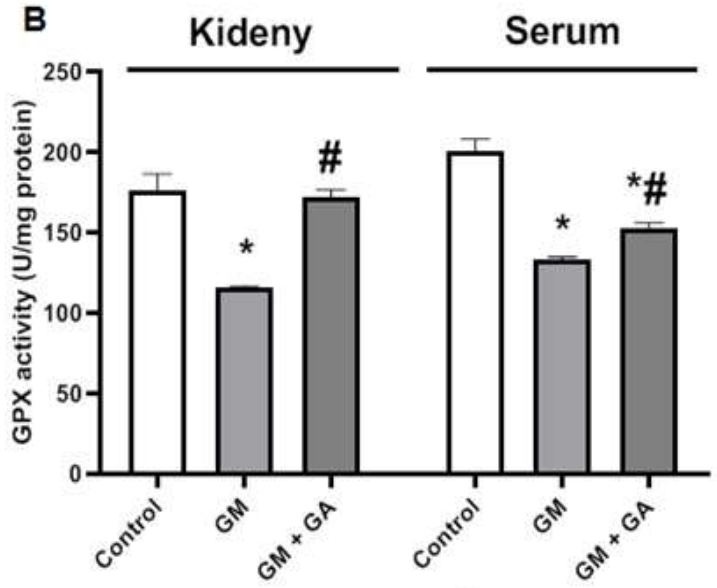
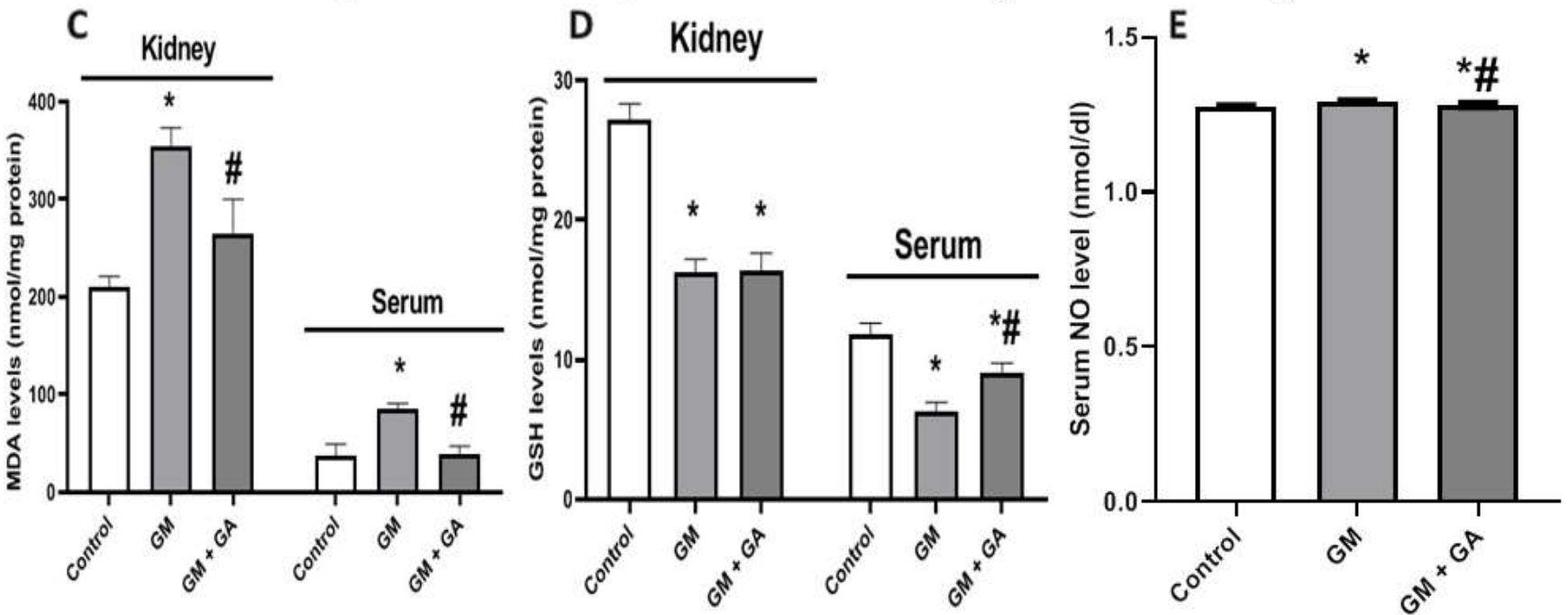

Figure 5. The effects of GA on the serum and renal levels of CAT (A) and GPX (B), MDA (C), and GSH (D) and the serum level of $N O(E)$ in GM nephrotoxicity in rats. Bars display mean \pm SEM. One-way ANOVA followed by a post hoc LSD test was applied for comparison between groups. ${ }^{*} P<0.05$ as compared with the control group. ${ }^{\#} P<0.05$ as compared with the GM group. 


\section{Gene Expression of Antioxidant Enzymes in the Kidney}

As depicted in Figure 6, significantly lower levels of mRNA expression of Cu-Zn SOD, CAT, and GPX (3-fold, 88.49-fold, and 62.5-fold, respectively) were found in untreated nephrotoxic rats after 12 days of GM administration than in control animals. Expression levels of Cu-Zn SOD, CAT, and GPX were also detected in the treated group, with their expressions being 2.51-fold, 37.22-fold, and 53-fold, respectively, higher in treated nephrotoxic animals than in untreated nephrotoxic rats.

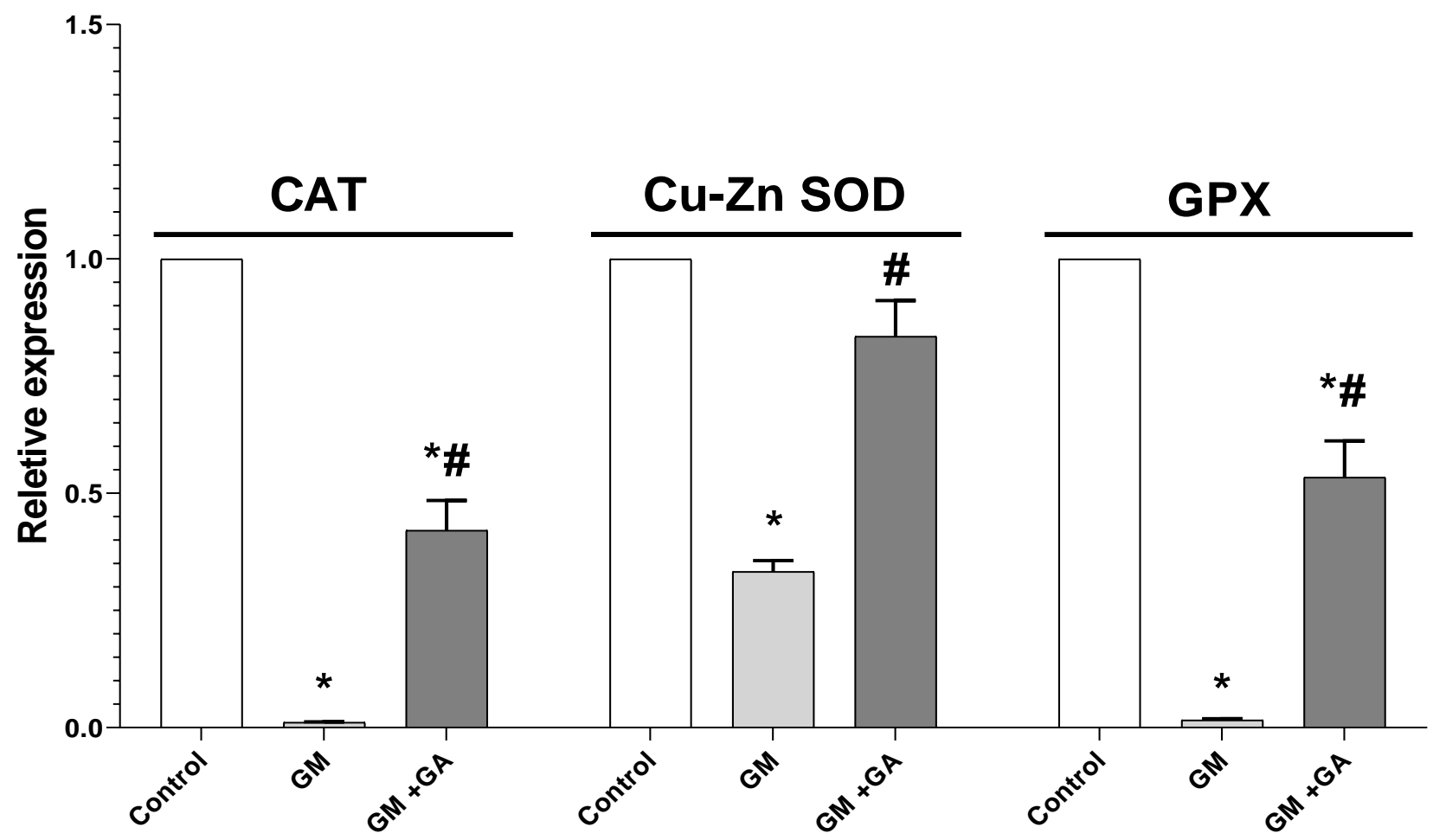

Figure 6. The effects of GA administration on the renal mRNA expression levels of antioxidant enzymes including CAT, $\mathrm{Cu}-\mathrm{Zn}$ SOD, and GPX in rats with GM nephrotoxicity. Bars indicate mean \pm SEM. One-way ANOVA followed by a post hoc Dunnett T3 test was applied for comparison between groups. ${ }^{*} P<0.05$ as compared with the control group. ${ }^{\#} P<0.05$ as compared with the GM group.

\section{DISCUSSION}

GM is an aminoglycoside antibiotic that is highly used to treat gram-negative bacterial infections. Unfortunately, the clinical application of this antibiotic is associated with severe side effects such as nephrotoxicity, which is observed in up to $20 \%$ therapeutic courses and confined to the utilization of the drug [36]. Several reports have shown that the applications of natural antioxidant ingredients are effective in reducing the side effects of GM, especially nephrotoxicity [37]. Here, we demonstrated the protective effects of a $100 \mathrm{mg} / \mathrm{kg}$ dose of natural antioxidant GA in a rodent model of GM nephrotoxicity. Our results revealed that 12 days of GA administration in combination with GM ameliorated GM induced renal histopathological damages and improved the biochemical parameters related to the renal and liver functions. Additionally, GA could antagonize GM effects on lipid profile and atherogenic indices. Finally, GA could reduce NO and MDA levels and increase GSH concentrations as well as the activities and gene expressions of antioxidant enzymes.

Renal function impairment due to GM was confirmed biochemically by determining the increased proteinuria excretion rate and serum levels of urea and $\mathrm{Cr}$. Additionally, it was also approved histopathologically by the observation of the high levels of tubular necrosis, eosinophilic cast, and leucocyte infiltration. All of these results were in accordance with the findings of previous studies [8]. Interestingly, the adverse effects of GM were significantly improved by 12 days of GA administration $(100 \mathrm{mg} / \mathrm{kg})$, showing that the nephroprotective effects of GA against GM nephrotoxicity. More recently, similar studies showed that GA in doses of $30,200,400 \mathrm{mg} / \mathrm{kg}$ administration for seven or eight consecutive days could improve renal histopathological lesions and kidney dysfunction in rats intoxicated with GM, likely through its antioxidant activities and its ability to maintain the cellular membrane integrity [38]. Also, previous studies indicated that GA could protect the kidney in pathological situations such as lindane induced toxicity $[39,40]$ and RIR [19] 
through its anti-oxidative properties. The results offered by a published study expressed that GA ( $20 \mathrm{mg} / \mathrm{kg}$, for seven days) isolated from Peltiphyllum peltatum mitigated the serum levels of urea, $\mathrm{Cr}$, uric acid, and blood urea nitrogen (BUN) in rats with NaF induced nephrotoxicity [41]. Moreover, the findings of our study have confirmed previous researches on the effects of other antioxidants in ameliorating kidney damages due to GM that can refer to aminoguanidine [42], gossypin [43], and nigella sativa [44]. Therefore, the protective roles of GA in reducing the levels of renal function markers and histopathological changes may be associated with its antioxidant activities, as reported in the present study and above-mentioned previous studies.

It has been demonstrated that GM could directly induce oxidative stress and apoptosis in the liver and finally result in hepatotoxicity $[45,46]$. Furthermore, several studies have indicated that acute kidney injuries (AKI) such as RIR and nephrotoxicity caused by cisplatin and GM can result in damage to other organs such as the liver $[47,48]$. The detailed mechanisms of AKI induced liver damage are well unknown. However, different agents such as uremic stress, ROS, inflammatory cytokines (TNF- $\alpha$, IL-17A, and IL-6), and peptidyl arginine deiminase 4 (PAD-4) have been recognized as the mediators of liver damages during AKI [49]. Khaksari and coauthors showed that GM directly enhanced apoptosis and oxidative stress in the liver of rats. Arjinajarn and coauthors [50] and Mohamadi Yarijani and coauthors [8] reported that GM induced increased AST, ALT, and ALP activities, elevated ROS and LPO productions, enhanced mitochondrial activated apoptosis and intensified liver histopathological damages. In our results, we similarly found a marked elevation in liver function parameters in GM treated rats, showing that liver injuries were induced during GM administration. Because these enzymes are present in the cytoplasm and are discharged into blood flow after altering the permeability of cellular membrane [51]. Previous studies declared that natural antioxidant compounds such as Malva sylvestris [8], palmatine [46] and vitamin E [49] could ameliorate the harmful effects of GM and AKI on the liver tissue. Interestingly, we found these serum activities of ALT, AST, and ALP significantly reduced in nephrotoxic rats after treatment with GA. As reported previously, the hepatoprotective effects of GA may be related to its anti-oxidative and anti-inflammatory characteristics in the kidney and its impact on the liver tissue [19]. Further investigations are suggested to determine this matter.

The findings of this study indicated that the levels of TG, Chol, VLDL, LDL, and atherogenic indices markedly augmented in the nephrotoxic rats. However, the level of HDL-C significantly reduced following GM treatment. Apart from TG and VLDL, treatment with GA could significantly reverse all of the above findings Furthermore, PON1 activity significantly declined in nephrotoxic rats, and treatment with GA could increase PON1 activity, but it was not statistically significant. PON1, as an esterase, prevents LDL peroxidation and the formation of oxidized LDL. It is stated that PON1 activity included positive and negative associations with HDL and atherogenic indices, respectively in GM nephrotoxicity. The decline of PON1 activity could relate to oxidative stress caused by GM similar to some reports [52]. Therefore, compounds with high antioxidant activity can amend lipid profile levels and also PON1 activity [52]. Hypolipidemic potentials of herbal antioxidants like GA may result in the prevention of lipoprotein metabolism pathways through the intercepting of enzymes and proteins function involved in the pathway. Additionally, antioxidants can reduce fat absorption, incite cholesterol secretion through bile, and enhance cholesterol excretion into feces [53,54]. Following the present study, previous studies illustrated hypolipidemic properties of other natural antioxidants such as oleuropein, histidine, the combination of quercetin and vitamin $\mathrm{C}$, and the combination of quercetin and $\alpha$-tocopherol $[52,55,56]$.

Our study results revealed that GM considerably augmented MDA and NO levels and also diminished GSH levels. In contrast, treatment with GA displayed inverse results from MDA, NO, and serum GSH levels. MDA is recognized as being an LPO marker and the last decomposition product of unsaturated fatty acids peroxides. One of the primary reasons for the enhanced LPO in the GM group may be GM induced oxidative stress in the cellular environment and the reduction of GSH that subsequently incremented MDA levels. In consistent with our findings, several reports highlighted that natural antioxidants such as eugenol, selenium, hydroxytyrosol, and quercetin included the ameliorative effects on MDA and GSH levels [57].

Moreover, other observations indicated that GA could mitigate LPO and GSH levels in RIR and NaF nephrotoxicity [41]. NO is a potent vasodilator that physiologically regulates the vascular system and renal tubular function $[19,58]$. Increased NO level plays the primary role in tissue damage through the reaction with superoxide anion and the production of peroxynitrite as a powerful oxidant. Our results share several similarities with the findings of Ghaznavi and coauthors [38], showing that GA and other natural antioxidants could reduce LPO and NO and increase GSH content.

In our study, the serum and renal activities of CAT and GPX remarkably reduced in the nephrotoxic rats in comparison with the control rats, representing GM promoted the production ROS in the nephrotoxicity 
process and inactivated the function of antioxidant enzymes according to previous studies [59]. Additionally, renal mRNA expression levels of CAT, Cu-Zn SOD, and GPX significantly decreased after GM administration in nephrotoxic rats. By contrast, the treatment of nephrotoxic rats with GA could substantially prevent GM's effects on both activities and mRNA expression levels of antioxidant enzymes. Increased GPX and CAT activities observed in GA treated animals may be associated with GA abilities to scavenge ROS and increase gene expression levels of antioxidant enzymes [38]. In agreement with our study, the number of antioxidants confirmed our findings. For example, the antioxidants such as quercetin, lycopene, curcumin, and melatonin appear to be well in the improvement of oxidative stress status [20]. Moreover, different studies showed that GA could enhance the activities of antioxidant enzymes SOD, CAT, GPX, and GSH in many pathological conditions such as myocardial infarction, $\mathrm{CCl}$-induced chronic liver injury, diabetes, and spinal cord injury [60].

\section{CONCLUSION}

Our biochemical, histopathological, and molecular results showed that GA, at a dose of $100 \mathrm{mg} / \mathrm{kg} / \mathrm{day}$, has potential nephroprotective effects. Therefore, it has ameliorated GM nephrotoxicity in experimental rats.

Funding: This research received no external funding.

Acknowledgments: The authors would like to thank the officials of Lorestan University of Medical Sciences, Khorramabad, Iran for their support.

Conflicts of Interest: The authors declare no conflict of interest.

\section{REFERENCES}

1. Salgado C, Hernandes F, Novoa J. Glomerular nephrotoxicity of amino nucleosides. Toxicol Appl Pharmacol. 2007;223:86-98.

2. Lopez-Novoa JM, Quiros Y, Vicente L, Morales Al, Lopez-Hernandez FJ. New insights into the mechanism of aminoglycoside nephrotoxicity: an integrative point of view. Kidney Int. 2011;79(1):33-45.

3. Siegenthaler WE, Bonetti A, Luthy R. Aminoglycoside antibiotics in infectious diseases: an overview. Am J Med. 1986;80(6):2-14.

4. Balakumar P, Rohilla A, Thangathirupathi A. Gentamicin-induced nephrotoxicity: do we have a promising therapeutic approach to blunt it? Pharmacol Res. 2010;62(3):179-86.

5. Balakumar P, Chakkarwar VA, Kumar V, Jain A, Reddy J, Singh M. Experimental models for nephropathy. J Renin Angiotensin Aldosterone Syst. 2008;9(4):189-95.

6. Sener G, Sehirli AÖ, Altunbas HZ, Ersoy Y, Paskaloglu K, Arbak S, et al. Melatonin protects against gentamicininduced nephrotoxicity in rats. J Pineal Res. 2002;32(4):231-6.

7. Nitha B, Janardhanan K. Aqueous-ethanolic extract of morel mushroom mycelium Morchella esculenta, protects cisplatin and gentamicin induced nephrotoxicity in mice. Food Chem Toxicol. 2008;46(9):3193-9.

8. Mohamadi Yarijani Z, Najafi H, Shackebaei D, Madani SH, Modarresi M, Jassemi SV. Amelioration of renal and hepatic function, oxidative stress, inflammation and histopathologic damages by Malva sylvestris extract in gentamicin induced renal toxicity. Biomedicine \& pharmacotherapy = Biomedecine \& pharmacotherapie. 2019;112:108635.

9. Wang C, Salahudeen AK, McClain wttaoM, Whitehead J. Lipid peroxidation accompanies cyclosporine nephrotoxicity: Effects of vitamin E. Kidney Int. 1995;47(3):927-34.

10. Rao AS, Reddy SG, Babu PP, Reddy AR. The antioxidant and antiproliferative activities of methanolic extracts from Njavara rice bran. BMC complementary and alternative medicine. 2010;10:4.

11. Sadeeshkumar V, Arul D, Ravichandran S. Protective effects of gallic acid in the renal markers, histopathology and immunohistochemical studies on vancomycin induced nephrotoxic rats. Int J Adv Life Sci 2013;6(3):356-65.

12. Badhani B, Sharma N, Kakkar R. Gallic acid: a versatile antioxidant with promising therapeutic and industrial applications. Rsc Advances. 2015;5(35):27540-57.

13. Locatelli C, Filippin-Monteiro FB, Centa A, Creczinsky-Pasa TB. Handbook on Gallic Acid: Natural Occurrences, Antioxidant Properties and Health Implications (Biochemistry Research Trends), (4th Ed.). Nova Science Pub Inc 2013:1-23.

14. Cholbi M, Paya M, Alcaraz M. Inhibitory effects of phenolic compounds on CCl 4-induced microsomal lipid peroxidation. Cell Mol Life Sci. 1991;47(2):195-9.

15. Peng C-C, Hsieh C-L, Wang H-E, Chung J-Y, Chen K-C, Peng RY. Ferulic acid is nephrodamaging while gallic acid is renal protective in long term treatment of chronic kidney disease. Clin Nutr. 2012;31(3):405-14. 
16. Reckziegel P, Dias VT, Benvegnú DM, Boufleur N, Barcelos RCS, Segat HJ, et al. Antioxidant protection of gallic acid against toxicity induced by $\mathrm{Pb}$ in blood, liver and kidney of rats. Toxicol Rep. 2016;3:351-6.

17. Canbek M, Ustuuml MC, Kabay S, Uysal O, Ozden H, Sentuuml H, et al. The effect of gallic acid on kidney and liver after experimental renal ischemia/reperfusion injury in the rats. Afr J Pharm Pharmacol. 2011;5(8):1027-33.

18. Niho N, Shibutani M, Tamura T, Toyoda K, Uneyama C, Takahashi N, et al. Subchronic toxicity study of gallic acid by oral administration in F344 rats. Food Chem Toxicol. 2001;39(11):1063-70.

19. Ahmadvand H, Yalameha B, Adibhesami G, Nasri M, Naderi N, Babaeenezhad E, et al. The Protective Role of Gallic Acid Pretreatment On Renal Ischemia-reperfusion Injury in Rats. Reports of Biochemistry and Molecular Biology. 2019; 8(1):42-8.

20. Karahan I, Atessahin A, Yilmaz S, Ceribasi AO, Sakin F. Protective effect of lycopene on gentamicin-induced oxidative stress and nephrotoxicity in rats. Toxicology. 2005;215(3):198-204.

21. Lamarca B. Endothelial dysfunction; an important mediator in the Pathophysiology of Hypertension during Preeclampsia. Minerva ginecologica. 2012;64(4):309-20.

22. Shahangian S, Brown PI, Ash KO. Turbidimetric measurement of total urinary proteins: a revised method. Am J Clin Pathol. 1984;81(5):651-4.

23. Ahmadvand $\mathrm{H}$, Ghasemi-Dehnoo M. Antiatherogenic, hepatoprotective, and hypolipidemic effects of coenzyme Q10 in alloxan-induced type 1 diabetic rats. ARYA Atheroscler. 2014;10(4):192-8.

24. Cheraghi M, Ahmadvand H, Maleki A, Babaeenezhad E, Shakiba S, Hassanzadeh F. Oxidative Stress Status and Liver Markers in Coronary Heart Disease. Rep Biochem Mol Biol. 2019;8(1):49-55.

25. Friedewald WT, Levy RI, Fredrickson DS. Estimation of the concentration of low-density lipoprotein cholesterol in plasma, without use of the preparative ultracentrifuge. Clin Chem. 1972;18(6):499-502.

26. Ikewuchi C. Alteration of plasma lipid profiles and atherogenic indices by Stachytarpheta jamaicensis L.(Vahl). Biokemistri. 2009;21(2):71-7.

27. Ahmadvand H, Noori A, Dehnoo MG, Bagheri S, Cheraghi RA. Hypoglycemic, hypolipidemic and antiatherogenic effects of oleuropein in alloxan-induced Type 1 diabetic rats. Asian Pac J Trop Dis. 2014;4:S421-S5.

28. Ohkawa H, Ohishi N, Yagi K. Assay for lipid peroxides in animal tissues by thiobarbituric acid reaction. Analytical biochemistry. 1979;95(2):351-8.

29. Nasiry D, Khalatbary AR, Ahmadvand H, Talebpour Amiri F, Akbari E. Protective effects of methanolic extract of Juglans regia L. leaf on streptozotocin-induced diabetic peripheral neuropathy in rats. BMC complementary and alternative medicine. 2017;17(1):476.

30. Ellman GL. Tissue sulfhydryl groups. Arch Biochem Biophys. 1959;82(1):70-7.

31. Rotruck JT, Pope AL, Ganther H, Swanson A, Hafeman DG, Hoekstra W. Selenium: biochemical role as a component of glutathione peroxidase. Science. 1973:588-90.

32. Sinha AK. Colorimetric assay of catalase. Analytical biochemistry. 1972;47(2):389-94.

33. Ahmadvand H, Babaeenezhad E, Nasri M, Jafaripour L, Mohammadrezaei Khorramabadi R. Glutathione ameliorates liver markers, oxidative stress and inflammatory indices in rats with renal ischemia reperfusion injury. J Renal Inj Prev. 2018;8:91-7.

34. Giustarini D, Rossi R, Milzani A, Dalle-Donne I. Nitrite and nitrate measurement by Griess reagent in human plasma: evaluation of interferences and standardization. Methods Enzymol. 2008;440:361-80.

35. Jamor $\mathrm{P}$, Ahmadvand $\mathrm{H}$, Ashoory $\mathrm{H}$, Babaeenezhad $\mathrm{E}$. Effect of alpha-lipoic acid on antioxidant gene expression and kidney injury in alloxan-induced diabetic rats. J Nephropathol. 2019;8(1):e8.

36. Al-Shabanah OA, Aleisa AM, AI-Yahya AA, Al-Rejaie SS, Bakheet SA, Fatani AG, et al. Increased urinary losses of carnitine and decreased intramitochondrial coenzyme $A$ in gentamicin-induced acute renal failure in rats. Nephrol Dial Transplant. 2010;25(1):69-76.

37. Ali BH. Agents ameliorating or augmenting experimental gentamicin nephrotoxicity: some recent research. Food Chem Toxicol. 2003;41(11):1447-52.

38. Ghaznavi H, Fatemi I, Kalantari H, Hosseini Tabatabaei SMT, Mehrabani M, Gholamine B, et al. Ameliorative effects of gallic acid on gentamicin-induced nephrotoxicity in rats. Journal of Asian natural products research. 2018;20(12):1182-93.

39. Manshare K, Anand A, Mahajan S, Satija S, Sharma N, Khurana N, et al. Evaluation of Nephroprotective Activity of Gallic Acid in Gentamicin-induced rat Model of Nephrotoxicity. International Journal of Green Pharmacy. 2018;Special Issue:48-52.

40. Said MM. The protective effect of eugenol against gentamicin-induced nephrotoxicity and oxidative damage in rat kidney. Fundamental \& clinical pharmacology. 2011;25(6):708-16. 
41. Nabavi SM, Habtemariam S, Nabavi SF, Sureda A, Daglia M, Moghaddam AH, et al. Protective effect of gallic acid isolated from Peltiphyllum peltatum against sodium fluoride-induced oxidative stress in rat's kidney. Molecular and cellular biochemistry. 2013;372(1-2):233-9.

42. Polat A, Parlakpinar H, Tasdemir S, Colak C, Vardi N, Ucar M, et al. Protective role of aminoguanidine on gentamicin-induced acute renal failure in rats. Acta histochemica. 2006;108(5):365-71.

43. Katary M, Salahuddin A. Ameliorative effect of gossypin against gentamicin-induced nephrotoxicity in rats. Life sciences. 2017;176:75-81.

44. Yaman I, Balikci E. Protective effects of nigella sativa against gentamicin-induced nephrotoxicity in rats. Experimental and toxicologic pathology : official journal of the Gesellschaft fur Toxikologische Pathologie. 2010;62(2):183-90.

45. Alkahtani S, Alarifi SA, Al-Doaiss AA. Detection of apoptosis induced by gentamicin in rat hepatocytes. Int $\mathrm{J}$ Zool Res. 2009;5(4):161-70.

46. Khaksari M, Esmaili S, Abedloo R, Khastar H. Palmatine ameliorates nephrotoxicity and hepatotoxicity induced by gentamicin in rats. Arch Biochem Biophys. 2019:1-6.

47. Mohamadi Yarijani Z, Godini A, Madani SH, Najafi H. Reduction of cisplatin-induced renal and hepatic side effects in rat through antioxidative and anti-inflammatory properties of Malva sylvestris L. extract. Biomedicine \& pharmacotherapy = Biomedecine \& pharmacotherapie. 2018;106:1767-74.

48. Gardner DS, De Brot S, Dunford LJ, Grau-Roma L, Welham SJ, Fallman R, et al. Remote effects of acute kidney injury in a porcine model. American journal of physiology Renal physiology. 2016;310(4):F259-71.

49. Lee SA, Cozzi M, Bush EL, Rabb H. Distant Organ Dysfunction in Acute Kidney Injury: A Review. American journal of kidney diseases : the official journal of the National Kidney Foundation. 2018;72(6):846-56.

50. Arjinajarn P, Chueakula N, Pongchaidecha A, Jaikumkao K, Chatsudthipong V, Mahatheeranont S, et al. Anthocyanin-rich Riceberry bran extract attenuates gentamicin-induced hepatotoxicity by reducing oxidative stress, inflammation and apoptosis in rats. Biomedicine \& pharmacotherapy = Biomedecine \& pharmacotherapie. 2017;92:412-20.

51. Kalkan Y, Kapakin KA, Kara A, Atabay T, Karadeniz A, Simsek N, et al. Protective effect of Panax ginseng against serum biochemical changes and apoptosis in kidney of rats treated with gentamicin sulphate. Journal of molecular histology. 2012;43(5):603-13.

52. Ahmadvand H, Bagheri S, Tamjidi-Poor A, Cheraghi M, Azadpour M, Ezatpour B, et al. Biochemical effects of oleuropein in gentamicin-induced nephrotoxicity in rats. ARYA Atheroscler. 2016;12(2):87-93.

53. Cuzzocrea S, Mazzon E, Dugo L, Serraino I, Di Paola R, Britti D, et al. A role for superoxide in gentamicin-mediated nephropathy in rats. European journal of pharmacology. 2002;450(1):67-76.

54. Harris CS, Beaulieu LP, Fraser MH, McIntyre KL, Owen PL, Martineau LC, et al. Inhibition of advanced glycation end product formation by medicinal plant extracts correlates with phenolic metabolites and antioxidant activity. Planta medica. 2011;77(2):196-204.

55. Askari G, Hajishafiee M, Ghiasvand R, Hariri M, Darvishi L, Ghassemi S, et al. Quercetin and vitamin C supplementation: effects on lipid profile and muscle damage in male athletes. International journal of preventive medicine. 2013;4(Suppl 1):S58-62.

56. Nasri M, Mahdavifard S, Babaeenezhad E, Adibhesami G, Nouryazdan N, Veiskarami S, et al. Ameliorative effects of histidine on oxidative stress, tumor necrosis factor alpha (TNF- $\alpha$ ), and renal histological alterations in streptozotocin/nicotinamide-induced type 2 diabetic rats. Iran J Basic Med Sci. 2020;23(6):714-23.

57. Chashmi NA, Emadi S, Khastar H. Protective effects of hydroxytyrosol on gentamicin induced nephrotoxicity in mice. Biochemical and biophysical research communications. 2017;482(4):1427-9.

58. Abd-Elhamid TH, Elgamal DA, Ali SS, Ali FEM, Hassanein EHM, El-Shoura EAM, et al. Reno-protective effects of ursodeoxycholic acid against gentamicin-induced nephrotoxicity through modulation of NF-kappaB, eNOS and caspase-3 expressions. Cell and tissue research. 2018;374(2):367-87.

59. Anandan R, Subramanian P. Renal protective effect of hesperidin on gentamicin-induced acute nephrotoxicity in male Wistar albino rats. Redox report : communications in free radical research. 2012;17(5):219-26.

60. Kahkeshani N, Farzaei F, Fotouhi M, Alavi SS, Bahramsoltani R, Naseri R, et al. Pharmacological effects of gallic acid in health and disease: A mechanistic review. Iran J Basic Med Sci. 2019;22(3):225-37.

(C) 2020 by the authors. Submitted for possible open access publication under the terms and conditions of the Creative Commons Attribution (CC BY NC) license (https://creativecommons.org/licenses/by-nc/4.0/). 